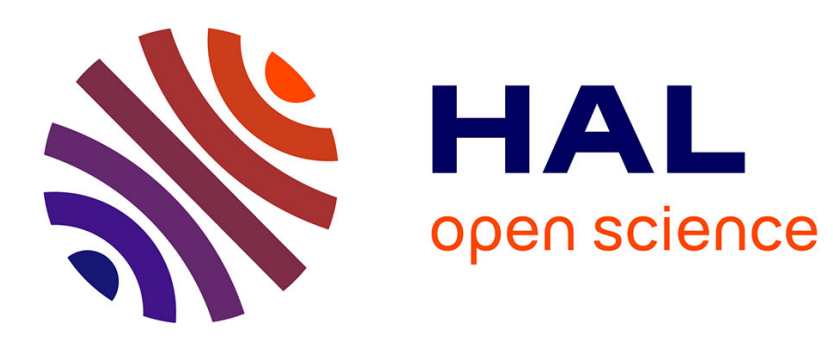

\title{
Implementation of the critical points model in a SFM-FDTD code working in oblique incidence
}

\author{
M. Hamidi, F.I. Baida, A. Belkhir, O. Lamrous
}

\section{To cite this version:}

M. Hamidi, F.I. Baida, A. Belkhir, O. Lamrous. Implementation of the critical points model in a SFM-FDTD code working in oblique incidence. Journal of Physics D: Applied Physics, 2011, 44 (24), pp.245101. 10.1088/0022-3727/44/24/245101 . hal-00605286

\section{HAL Id: hal-00605286 \\ https://hal.science/hal-00605286}

Submitted on 3 May 2021

HAL is a multi-disciplinary open access archive for the deposit and dissemination of scientific research documents, whether they are published or not. The documents may come from teaching and research institutions in France or abroad, or from public or private research centers.
L'archive ouverte pluridisciplinaire HAL, est destinée au dépôt et à la diffusion de documents scientifiques de niveau recherche, publiés ou non, émanant des établissements d'enseignement et de recherche français ou étrangers, des laboratoires publics ou privés. 


\title{
Implementation of the critical points model in a SFM-FDTD code working in oblique incidence
}

\author{
M Hamidi ${ }^{1}$, F I Baida ${ }^{2}$, A Belkhir ${ }^{1}$ and O Lamrous ${ }^{1}$ \\ ${ }^{1}$ Laboratoire de Physique et Chimie Quantique, Université Mouloud Mammeri, Tizi-Ouzou, Algeria \\ 2 Département d'Optique P.M. Duffieux, Institut FEMTO-ST UMR 6174 CNRS Université de \\ Franche-Comté, 25030 Besançon Cedex, France \\ E-mail: omarlamrous@mail.ummto.dz
}

\begin{abstract}
We describe the implementation of the critical points model in a finite-difference-time-domain code working in oblique incidence and dealing with dispersive media through the split field method. Some tests are presented to validate our code in addition to an application devoted to plasmon resonance of a gold nanoparticles grating.
\end{abstract}

\section{Introduction}

The description of permittivity of metals has been the focus of investigations from both the theoretical and experimental points of view due to their possible application in the nanooptic domain [1-5]. In particular, nano-optical periodic structures are usually composed of metal parts which present large dispersion over the range of wavelengths of interest. In this context, the calculation of their optical response spectra remains difficult when these structures are illuminated at oblique incidence which breaks the symmetry of the problem. Consequently, in our initial study, we have extended the finitedifference time-domain (FDTD) algorithm to work in oblique incidence [6]. To take into account the metal dispersion, implementation of split field method (SFM) with integration of the metal dispersion considering the Debye, Drude and DrudeLorentz models has been realized [7,8]. Recently, a new analytical model called the critical points (CPs) model $[1,2]$ has been used to describe more accurately the permittivity of several metals $[9,10]$. Its implementation in the FDTD algorithm using the recursive convolution (RC) method, has underlined a better description of the permittivity than the Drude-Lorentz model, over a wider range of wavelengths [11]. However, let us note that the proposed technique has been developed only for normal incidence case.

For this work, our aim is to extend the implementation of the SFM with integration of the metal dispersion considering the CP model. Thus, to the best of our knowledge, and in contrast to [9-11], this is the first time that the CP model dispersion has been implemented in oblique incidence.

The outline of this paper is as follows: the implementation of the CP model developed is presented in section 2. A summary of the SFM method already detailed in [6] is recalled and followed by a description of the calculation procedure of the electromagnetic field components. The simulation results of one-(1D) and three-dimensional (3D) gold periodic structures are illustrated in section 3 . We focus the analysis on the variation of the transmission versus the wavelengths and the incidence angles. We also show the possibility to predict and optimize the extinction spectra of metallic nanostructures. The conclusion of the work is summarized in section 4 .

\section{Theoretical developments}

It is well known that the FDTD method is a useful numerical simulation technique for solving Maxwell's equations. However, as the traditional FDTD method is based on a temporal finite-difference algorithm, the consideration of the oblique incidence problem especially in the case of periodic structures is not possible; this is due to wave frequency terms appearing in the periodic conditions. In accordance with these conditions, the problem is efficiently solved through the replacement of the electromagnetic fields $\vec{E}$ and $\vec{H}$ by the new variables $\vec{P}$ and $\vec{Q}$ expressed as

$$
\begin{aligned}
& \vec{P}=\vec{E} \mathrm{e}^{-\mathrm{i}\left(k_{x} x+k_{y} y\right)}, \\
& \vec{Q}=\vec{H} \mathrm{e}^{-\mathrm{i}\left(k_{x} x+k_{y} y\right)} .
\end{aligned}
$$


Then, in the context of the SFM method [12], intermediate variables $\overrightarrow{P_{a}}$ and $\overrightarrow{Q_{a}}$ are introduced in order to obtain new Maxwell's equations for which the resolution is adapted to the Yee scheme. Therefore, the FDTD implementation is accomplished by the following equations which relate the intermediate variables to global ones [8]:

$$
\begin{aligned}
& \frac{\partial P_{x a}}{\partial t}=\frac{1}{\varepsilon}\left[\frac{\partial Q_{z}}{\partial y}-\frac{\partial Q_{y}}{\partial z}\right], \\
& \frac{\partial P_{y a}}{\partial t}=\frac{1}{\varepsilon}\left[\frac{\partial Q_{x}}{\partial z}-\frac{\partial Q_{z}}{\partial x}\right], \\
& \frac{\partial P_{z a}}{\partial t}=\frac{1}{\varepsilon}\left[\frac{\partial Q_{y}}{\partial x}-\frac{\partial Q_{x}}{\partial y}\right], \\
& \frac{\partial Q_{x a}}{\partial t}=\frac{1}{\mu}\left[\frac{\partial P_{y}}{\partial z}-\frac{\partial P_{z}}{\partial y}\right], \\
& \frac{\partial Q_{y a}}{\partial t}=\frac{1}{\mu}\left[\frac{\partial P_{z}}{\partial x}-\frac{\partial P_{x}}{\partial z}\right], \\
& \frac{\partial Q_{z a}}{\partial t}=\frac{1}{\mu}\left[\frac{\partial P_{x}}{\partial y}-\frac{\partial P_{y}}{\partial x}\right]
\end{aligned}
$$

and

$$
\begin{aligned}
P_{z} & =\frac{1}{1-\frac{k_{x}^{2}}{\varepsilon \mu \omega^{2}}-\frac{k_{y}^{2}}{\varepsilon \mu \omega^{2}}}\left[P_{z a}+\frac{k_{x}}{\varepsilon \omega} Q_{y a}-\frac{k_{y}}{\varepsilon \omega} Q_{x a}\right], \\
Q_{z} & =\frac{1}{1-\frac{k_{x}^{2}}{\varepsilon \mu \omega^{2}}-\frac{k_{y}^{2}}{\varepsilon \mu \omega^{2}}}\left[Q_{z a}+\frac{k_{y}}{\mu \omega} P_{x a}-\frac{k_{x}}{\mu \omega} P_{y a}\right], \\
P_{x} & =P_{x a}+\frac{k_{y}}{\varepsilon \omega} Q_{z}, \\
P_{y} & =P_{y a}-\frac{k_{x}}{\varepsilon \omega} Q_{z}, \\
Q_{x} & =Q_{x a}-\frac{k_{y}}{\mu \omega} P_{z}, \\
Q_{y} & =Q_{y a}+\frac{k_{x}}{\mu \omega} P_{z} .
\end{aligned}
$$

Let us mention that system (4) becomes useless in the case of normal incidence $\left(k_{x}=k_{y}=0\right)$. On the other hand, we need to know the explicit dependence of the permittivity $\varepsilon(\omega)$ describing the dielectric properties of the considered media. For our contribution, this is done by considering the CP model. Let us recall that this later model has been discussed in a recent study which describes well the optical properties of metals (gold, silver, etc) in the visible/near-uv region. $\varepsilon(\omega)$ may be expressed as follows:

$$
\varepsilon_{\mathrm{DCP}}=\varepsilon_{\infty}-\frac{\omega_{P}^{2}}{\omega^{2}+\mathrm{i} \gamma \omega}+\sum_{p=1}^{p=2} G_{p}(\omega),
$$

where

$$
G_{p}(\omega)=A_{p} \Omega_{p}\left(\frac{\mathrm{e}^{\mathrm{i} \phi_{p}}}{\Omega_{p}-\omega-\mathrm{i} \Gamma_{p}}+\frac{\mathrm{e}^{-\mathrm{i} \phi_{p}}}{\Omega_{p}+\omega+\mathrm{i} \Gamma_{p}}\right) .
$$

The two first terms of equation (5) denote the standard contribution of the Drude model where $\varepsilon_{\infty}$ is the highfrequency limit dielectric constant; $\omega_{P}$ and $\gamma$ characterize the plasma frequency and damping coefficient, respectively.
The sum in equation (5) represents the contribution of the inter-band transitions with $A_{p}, \Omega_{p}, \phi_{p}$ and $\Gamma_{p}$ corresponding to the amplitude, gap energy, phase and broadening in that order $[1,2]$. For our part, we made use of the auxiliary differential equation (ADE) method for implementing the $\mathrm{CP}$ model. Consequently, we introduce the electric displacement field $(\vec{D})$ expressed as

$$
\vec{D}=\varepsilon_{0} \varepsilon_{\mathrm{DCP}} \vec{E}
$$

In the case of the CP model, $\vec{D}$ can be written as the sum of two electric displacement fields corresponding to the two contributions in the permittivity expression:

$$
\vec{D}=\vec{D}_{D}+\sum_{p=1}^{2} \vec{D}_{C p}
$$

with

$$
\begin{aligned}
& \vec{D}_{D}=\varepsilon_{0}\left[\varepsilon_{\infty}-\frac{\omega_{P}^{2}}{\omega^{2}+\mathrm{i} \gamma \omega}\right] \vec{E} \\
& \vec{D}_{C p}=\varepsilon_{0}\left[A_{p} \Omega_{p}\left(\frac{\mathrm{e}^{\mathrm{i} \phi_{p}}}{\Omega_{p}-\omega-\mathrm{i} \Gamma_{p}}+\frac{\mathrm{e}^{-\mathrm{i} \phi_{p}}}{\Omega_{p}+\omega+\mathrm{i} \Gamma_{p}}\right)\right] \vec{E}
\end{aligned}
$$

Using these relationships, we deduce the following decoupled equations:

$$
\begin{gathered}
\left(\frac{\partial^{2}}{\partial t^{2}}+\gamma \frac{\partial}{\partial t}\right) \overrightarrow{D_{D}}=\varepsilon_{0} \varepsilon_{\infty}\left(\frac{\partial^{2}}{\partial t^{2}}+\gamma \frac{\partial}{\partial t}+\frac{\omega_{P}^{2}}{\varepsilon_{\infty}}\right) \vec{E} \\
\left(\Omega_{p}^{2}+\Gamma_{p}^{2}+\frac{\partial^{2}}{\partial t^{2}}+2 \Gamma_{p} \frac{\partial}{\partial t}\right) \overrightarrow{D_{C p}} \\
=2 \varepsilon_{0} A_{p} \Omega_{p}\left(\sqrt{\Gamma_{p}^{2}+\Omega_{p}^{2}} \sin \left(\theta_{p}-\phi_{p}\right)\right. \\
\left.-\sin \phi_{p} \frac{\partial}{\partial t}\right) \vec{E}
\end{gathered}
$$

where $\theta_{p}=\arctan \left(\Omega_{p} / \Gamma_{p}\right)$.

In the case of oblique incidence, the components $P_{x a}$, $P_{y a}, P_{z a}, P_{x}, P_{y}, P_{z}$ and $Q_{z}$ considered in the equations ( $3 a$ )(3f) and (4a)-(4f) require a special treatment insofar as they cannot be included in the FDTD algorithm due to the dispersion relation $\varepsilon(\omega)$. For this, we use a new set of variables expressed in a similar way to that of equation (7). We illustrate this procedure by considering for example the $x$ component for which the new introduced variable is $L_{x a}=\varepsilon_{0} \varepsilon_{\mathrm{DCP}} P_{x a}$. According to equation $(3 a)$ :

$$
\frac{\partial L_{x a}}{\partial t}=\left[\frac{\partial Q_{z}}{\partial y}-\frac{\partial Q_{y}}{\partial z}\right] .
$$

The discretization of this last equation allows us to calculate the $L_{x a}$ variable as follows:

$$
\begin{aligned}
& L_{x a}^{n+1}(i, j, k)=L_{x a}^{n}(i, j, k) \\
& \quad+\frac{\Delta t}{\Delta y}\left[Q_{z}^{n}(i, j, k)-Q_{z}^{n}(i, j-1, k)\right] \\
& \quad-\frac{\Delta t}{\Delta z}\left[Q_{y}^{n}(i, j, k)-Q_{y}^{n}(i, j, k-1)\right] .
\end{aligned}
$$


(a)

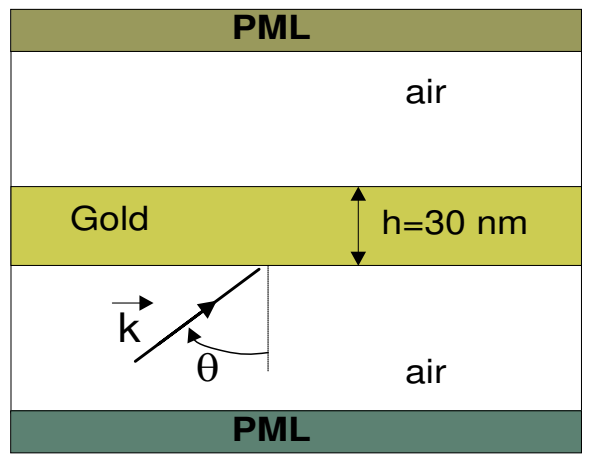

(b)
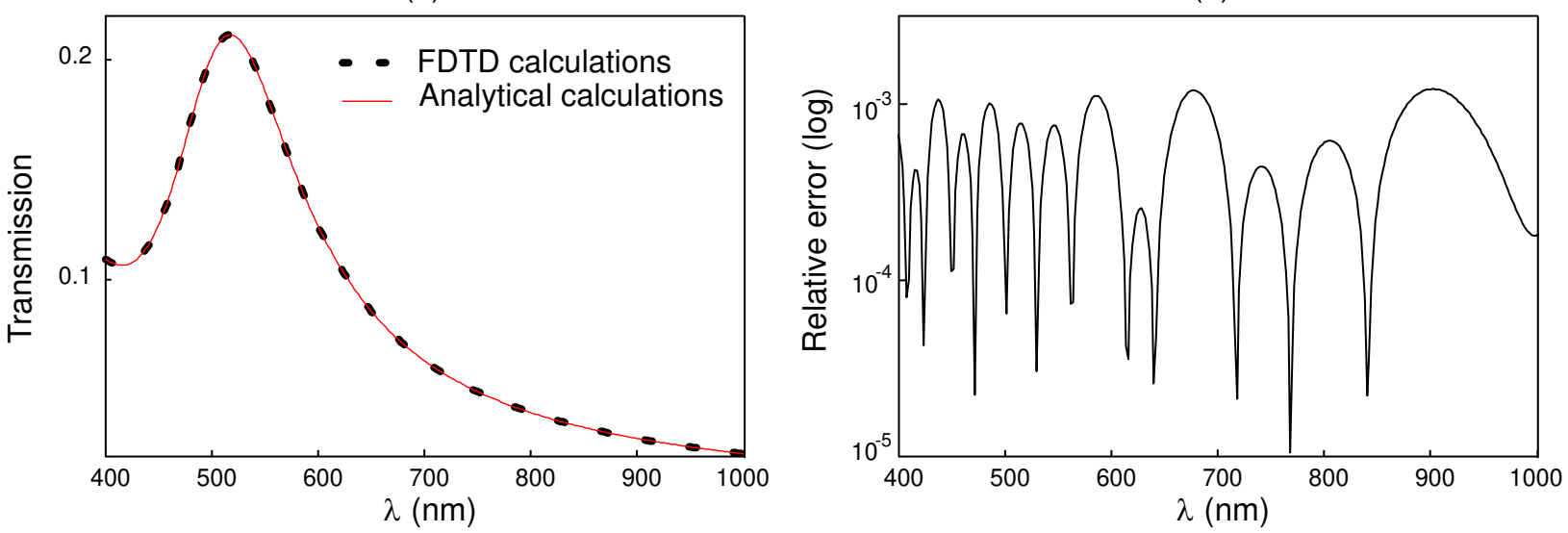

(c)

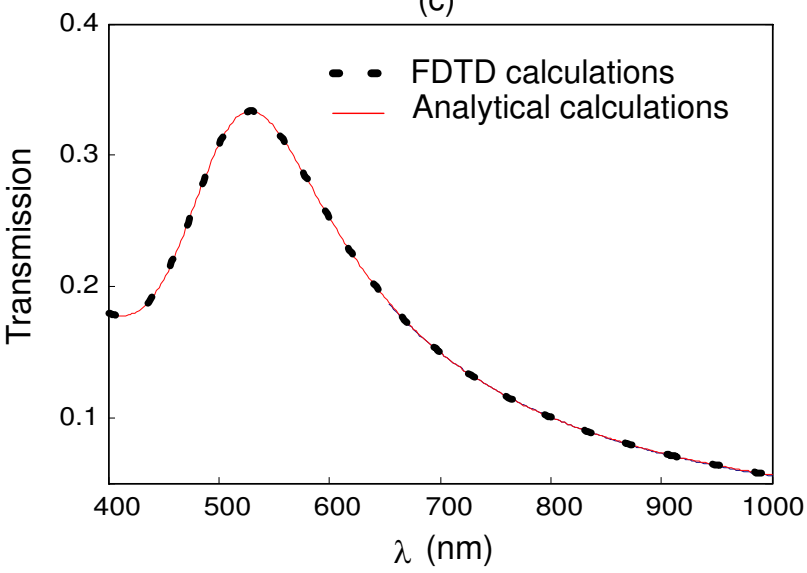

(e)

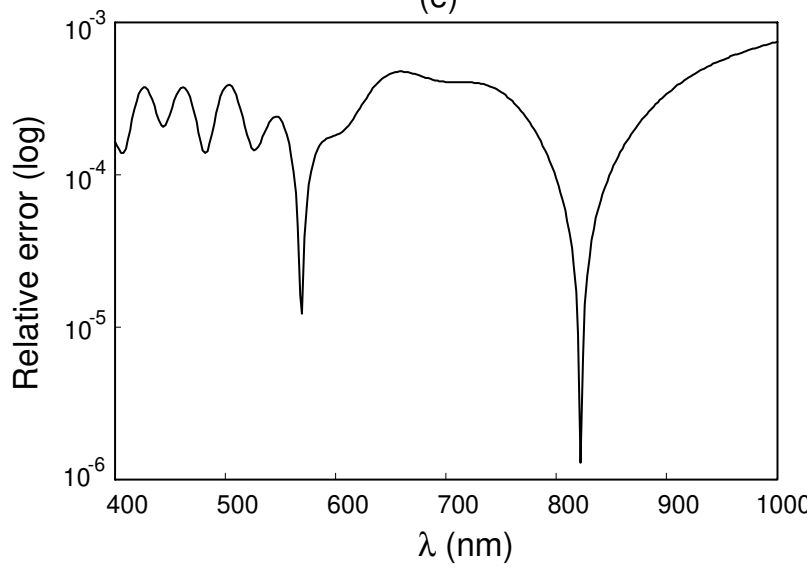

Figure 1. Transmission coefficient versus the wavelength for a $30 \mathrm{~nm}$ thick gold layer surrounded by air $(a)$ for TE $((b),(d))$ and TM polarization $((c),(e))$. Comparisons between analytical and FDTD calculations are presented in $(b)$ and $(c)$, while the relative errors are depicted in $(d)$ and $(e)$, respectively. The incident angle is fixed to $40^{\circ}$.

From equations (8), $(9 a)$ and $(9 b), \quad L_{x a}$ may be expressed as

$$
L_{x a}=L_{x a_{D}}+\sum_{p=1}^{p=2} L_{x a_{C p}} .
$$

with

$$
\begin{aligned}
& L_{x a_{D}}=\varepsilon_{0}\left[\varepsilon_{\infty}-\frac{\omega_{P}^{2}}{\omega^{2}+\mathrm{i} \gamma \omega}\right] P_{x a}, \\
& L_{x a_{C} p}=\varepsilon_{0}\left[A_{p} \Omega_{p}\left(\frac{\mathrm{e}^{\mathrm{i} \phi_{p}}}{\Omega_{p}-\omega-\mathrm{i} \Gamma_{p}}+\frac{\mathrm{e}^{-\mathrm{i} \phi_{p}}}{\Omega_{p}+\omega+\mathrm{i} \Gamma_{p}}\right)\right] P_{x a} .
\end{aligned}
$$

In this consideration, equations $(10 a)$ and $(10 b)$ become, respectively:

$$
\left(\frac{\partial^{2}}{\partial t^{2}}+\gamma \frac{\partial}{\partial t}\right) L_{x a_{D}}=\varepsilon_{0} \varepsilon_{\infty}\left(\frac{\partial^{2}}{\partial t^{2}}+\gamma \frac{\partial}{\partial t}+\frac{\omega_{P}^{2}}{\varepsilon_{\infty}}\right) P_{x a}
$$

$\left(\Omega_{p}^{2}+\Gamma_{p}^{2}+\frac{\partial^{2}}{\partial t^{2}}+2 \Gamma_{p} \frac{\partial}{\partial t}\right) L_{x a_{C p}}$

$$
=2 \varepsilon_{0} A_{p} \Omega_{p}\left(\sqrt{\Gamma_{p}^{2}+\Omega_{p}^{2}} \sin \left(\theta_{p}-\phi_{p}\right)-\sin \phi_{p} \frac{\partial}{\partial t}\right) P_{x a} .
$$


(a)

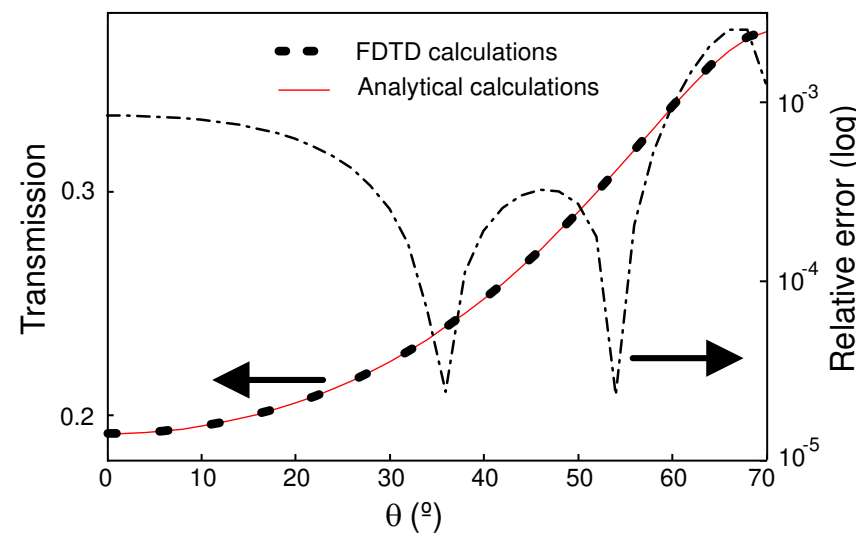

(b)

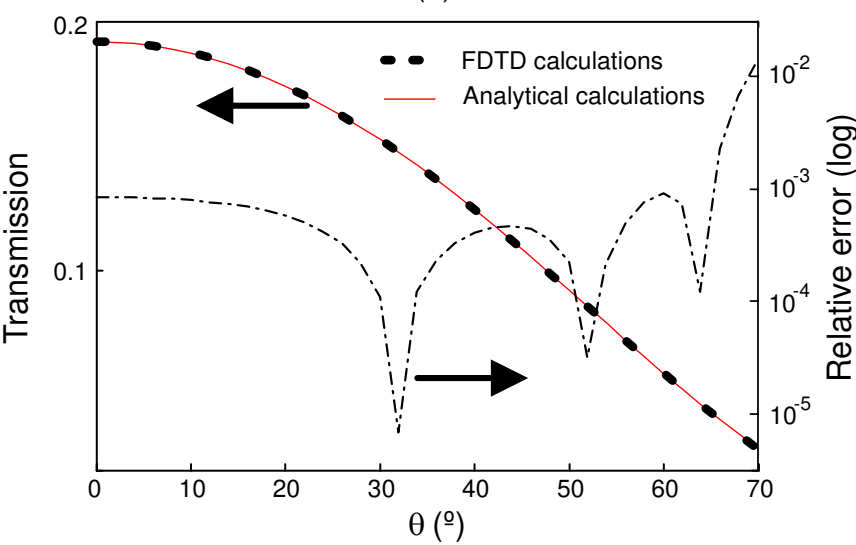

Figure 2. Transmission coefficient versus the angle of incidence for a $30 \mathrm{~nm}$ thick gold layer surrounded by air for TM $(a)$ and TE polarization $(b)$. Comparisons between analytical and FDTD calculations with the relative errors are presented. The wavelength is fixed to $600 \mathrm{~nm}$.

Finally, the partial derivatives of these last equations allow one to express the update equations:

$$
\begin{aligned}
P_{x a}^{n+1} & =\frac{1}{\frac{\chi_{D}}{\alpha_{D}}+\sum_{p=1}^{p=2}\left(\frac{x_{p}}{\alpha_{p}}\right)} \\
& \times\left[L_{x a}^{n+1}+\frac{\beta_{D}}{\alpha_{D}} L_{x a_{D}}^{n-1}+\frac{4}{\alpha_{D}} L_{x a_{D}}^{n}-\frac{\delta_{D}}{\alpha_{D}} P_{x a}^{n-1}-\frac{4 \varepsilon_{0} \varepsilon_{\infty}}{\alpha_{D}} P_{x a}^{n}\right. \\
& \left.+\sum_{p=1}^{p=2}\left(\frac{\beta_{p}}{\alpha_{p}} L_{x a_{C p}}^{n-1}-\frac{4}{\alpha_{p}} L_{x a_{C p}}^{n}\right)+\sum_{p=1}^{p=2}\left(\frac{\delta_{p}}{\alpha_{p}}\right) P_{x a}^{n-1}\right],
\end{aligned}
$$$$
L_{x a_{D}}^{n+1}=\frac{1}{\alpha_{D}}\left[-\beta_{D} L_{x a_{D}}^{n-1}-4 L_{x a_{D}}^{n}\right.
$$$$
\left.+\chi_{D} P_{x a}^{n-1}+\delta_{D} P_{x a}^{n-1}+4 \varepsilon_{0} \varepsilon_{\infty} P_{x a}^{n}\right],
$$$$
L_{x a_{C p}}^{n+1}=\frac{1}{\alpha_{p}}\left[-\beta_{p} L_{x a_{C p}}^{n-1}+4 L_{x a_{C p}}^{n}+\chi_{p} P_{x a}^{n+1}+\delta_{p} P_{x a}^{n-1}\right]
$$

with

$$
\begin{aligned}
& \alpha_{D}=-2-\gamma \Delta t, \\
& \beta_{D}=-2+\gamma \Delta t, \\
& \chi_{D}=\varepsilon_{0} \varepsilon_{\infty}\left[-2-\gamma \Delta t-\left(\omega_{p} \Delta t\right)^{2} / \varepsilon_{\infty}\right], \\
& \delta_{D}=\varepsilon_{0} \varepsilon_{\infty}\left[-2+\gamma \Delta t-\left(\omega_{p} \Delta t\right)^{2} / \varepsilon_{\infty}\right], \\
& \alpha_{p}=\left[\Omega_{p}^{2}+\Gamma_{p}^{2}\right] \Delta t^{2}+2 \Gamma_{p} \Delta t+2, \\
& \beta_{p}=\left[\Omega_{p}^{2}+\Gamma_{p}^{2}\right] \Delta t^{2}-2 \Gamma_{p} \Delta t+2, \\
& \chi_{p}=2 A_{p} \Omega_{p} \varepsilon_{0}\left[\Delta t^{2} \sqrt{\Omega_{p}^{2}+\Gamma_{p}^{2}} \sin \left(\theta_{p}-\phi_{p}\right)-\Delta t \sin \phi_{p}\right], \\
& \delta_{p}=2 A_{p} \Omega_{p} \varepsilon_{0}\left[\Delta t^{2} \sqrt{\Omega_{p}^{2}+\Gamma_{p}^{2}} \sin \left(\theta_{p}-\phi_{p}\right)+\Delta t \sin \phi_{p}\right] .
\end{aligned}
$$

Let us quote that the other components $P_{y a}, P_{z a}, P_{x}, P_{y}$, $P_{z}$ and $Q_{z}$ are calculated by following the same procedure.

\section{Numerical results: validation and application}

In this section, we present the computational results in order to validate and check the accuracy of our SFM-FDTD simulation

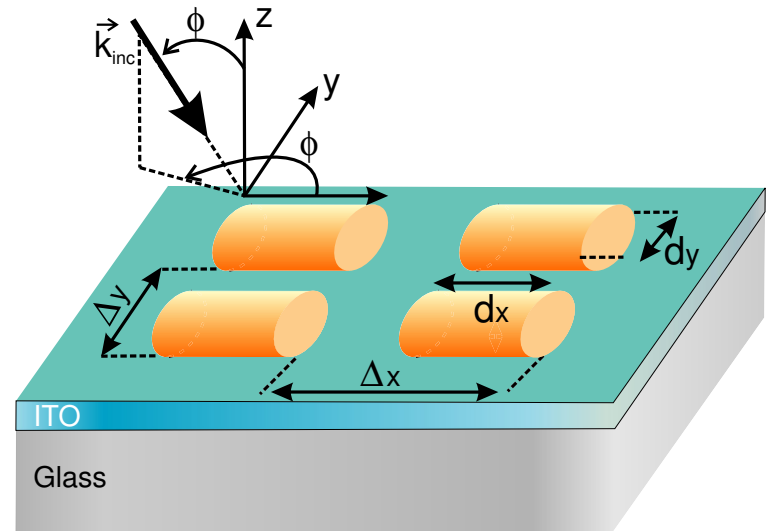

Figure 3. Geometry of the 3D structure studied for our calculations: $d_{x}=210 \mathrm{~nm}, d_{y}=110 \mathrm{~nm}, \Lambda_{X}=325 \mathrm{~nm}$ and $\Lambda_{Y}=310 \mathrm{~nm}$. The gold nanoparticle has a height of $h=45 \mathrm{~nm}$.

code. Let us mention that the parameters of the CP model are the same in [9] which present good agreement with the experimental data reported in [5]. In addition, to solve the boundary conditions in the $z$ direction the PML technique has been included according to $[6,8]$.

Figure 1(a) shows the geometry of the considered 1D structure which consists of the gold layer of $30 \mathrm{~nm}$ thickness surrounded by air. Figures $1(b)$ and $(c)$ illustrate both the FDTD and analytical transmission variation through this structure in TE and TM polarizations, respectively. The results presented here were obtained in the wavelength interval 400$1000 \mathrm{~nm}$ and for $\theta=40^{\circ}$. The comparison between our FDTD results and those obtained by analytical calculations shows qualitatively the same behaviour. In both cases of polarization, the relative error calculated as $\left(\left|T_{\mathrm{FDTD}}-T_{\text {analytical }}\right|\right) / T_{\text {analytical }}$ does not exceed $0.12 \%$ (see figures $1(d)$ and $(e)$ ). Consequently, good agreement between the two methods is achieved.

Figure 2 illustrates the transmission of the TE and TM polarizations versus the incidence angle at $600 \mathrm{~nm}$ wavelength. The results presented here were obtained in the angle incidence 

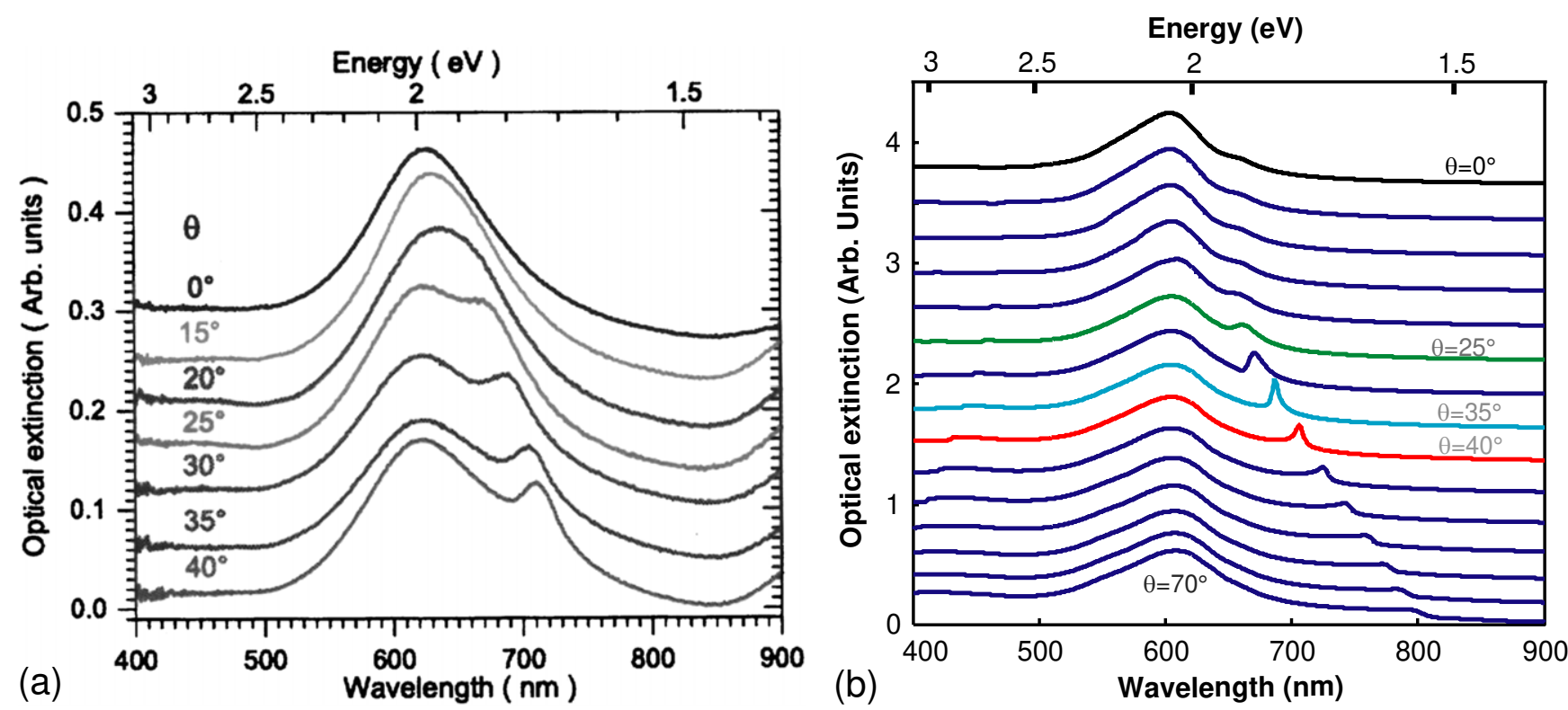

Figure 4. Optical extinction spectra versus incidence angle: experiment results of sample B in [14] (a) and FDTD results $(b)$ with TE polarization (parallel to $Y$ ).

interval such as $0 \leqslant \theta \leqslant 70^{\circ}$ in accordance with the computing and stability criterion $[6,8,13]$. Let us note that for $\theta>70^{\circ}$, the time step decreases so much that the computing time becomes very huge. Moreover, since the nanooptical experiments are frequently conducted for $\theta<60^{\circ}$, the considered interval is sufficient to validate the accuracy of our algorithm. As shown in figure 2, the theoretical curves fit our calculation results as well as for TE and TM polarizations. We observe that the relative error is less than $0.25 \%$ and $1.5 \%$ in the TM and TE polarization, respectively. At $\theta<60^{\circ}$, we observe a decrease in relative error under $0.1 \%$ denoting the validity and the accuracy of our code.

The 3D study deals with surface-enhanced Raman scattering through a metallic grating made in gold. The considered bi-periodic structure was experimentally studied by Félidj et al [14]. The structure consists on arrays of gold nanoparticles on top of a $30 \mathrm{~nm}$ indium-tin-oxide (ITO) coated glass substrate. The nanoparticles (sample B of [14]) are of prolate form (diameter $d_{x}=210 \mathrm{~nm}, d_{y}=110 \mathrm{~nm}$ and height $h=45 \mathrm{~nm}$ ). They are arranged in rectangular array with $x$ grating period (parallel to $X$ ) set to $\Lambda_{X}=325 \mathrm{~nm}$ and $y$ grating period (parallel to $Y$ ) set to $\Lambda_{Y}=310 \mathrm{~nm}$. Figure 4(a) shows the optical extinction spectra experimentally obtained for different incidence angles with incident plane waves linearly polarized along the $Y$ direction, i.e. TE polarization [14]. In order to compare our results with these experimental spectra, we define an FDTD similar structure of prolate particles of gold deposited on a $30 \mathrm{~nm}$ thick layer of ITO on the top of a glass substrate (see figure 3 ). The incident medium is the vacuum $\left(n_{1}=1\right)$ and the light transmission (the diffracted zero-order) is determined in the glass $\left(n_{2}=1.53\right)$. The ITO refractive index is fixed to $n=2$. The transmission is calculated for different values of the incidence angle $\theta$ and then the extinction spectra plotted versus the wavelength. Figure $4(b)$ shows our simulation spectra for $0<\theta<70^{\circ}$ with a step of $5^{\circ}$. One can note good agreement with those experimentally illustrated in [14]. A plasmon resonance is detected at $605 \mathrm{~nm}$ and, with regard to the experimental results $\left(\lambda_{\mathrm{SP}}=620 \mathrm{~nm}\right)$, the relative error is around $2 \%$. When considering the hardness to approach the experimental conditions, this relative error could be considered as fair to validate our code. As predicted by theory $[15,16]$, figures $4(a)$ and $(b)$ also show a plasmon resonance slight shift to the red upon appearance of the first grating order. In Lamprecht et al [17], this red-shift appears when the grating constant approaches the plasmon resonance wavelength. Upon a critical incident angle $\theta_{\mathrm{C}}$, the first grating order starts to radiate as shown by our simulation results. This critical angle is given by

$$
\theta_{\mathrm{C}}=\arcsin \left[\frac{1}{n_{1}}\left(\frac{\lambda_{\mathrm{SP}}}{\Lambda_{X}}-n_{2}\right)\right] .
$$

In our calculations (for $\lambda_{\mathrm{SP}}=605 \mathrm{~nm}$ ), we obtain $\theta_{\mathrm{C}} \approx 20^{\circ}$ slightly different from experimental results $\left(\theta_{\mathrm{C}} \approx 22^{\circ}\right)$ because of the difference between the two resonance wavelengths. At $\theta=40^{\circ}$, the extinction spectrum shows two peaks which occur at 605 and $706 \mathrm{~nm}$ (see figure 4(b)). Maps of electric field amplitude calculated in the $X Y, X Z$ and $Y Z$ planes for these resonance wavelengths are illustrated in figures 5 and figure 6. Let us mention that the $X Y$ plane is carried out into ITO layer for $10 \mathrm{~nm}$ under the gold nanostructure. As one can note it, there is significant change in the field repartition when comparing figures 5 and 6 . For $\lambda=605 \mathrm{~nm}$, the plasmon resonance depicted is due to the collective oscillations of electrons in the gold. This mode corresponds to the plasmon resonance of an isolated particle and it is illustrated by localization of the electric field only around each particle. On the other hand, for $\lambda=706 \mathrm{~nm}$, the corresponding peak could be attributed to the grating induced mode because the radiated light field is strongly coupled to the plasmon fields of neighbouring particles leading to a mixed light plasmon mode of the whole array. This is illustrated in figure 6 where 

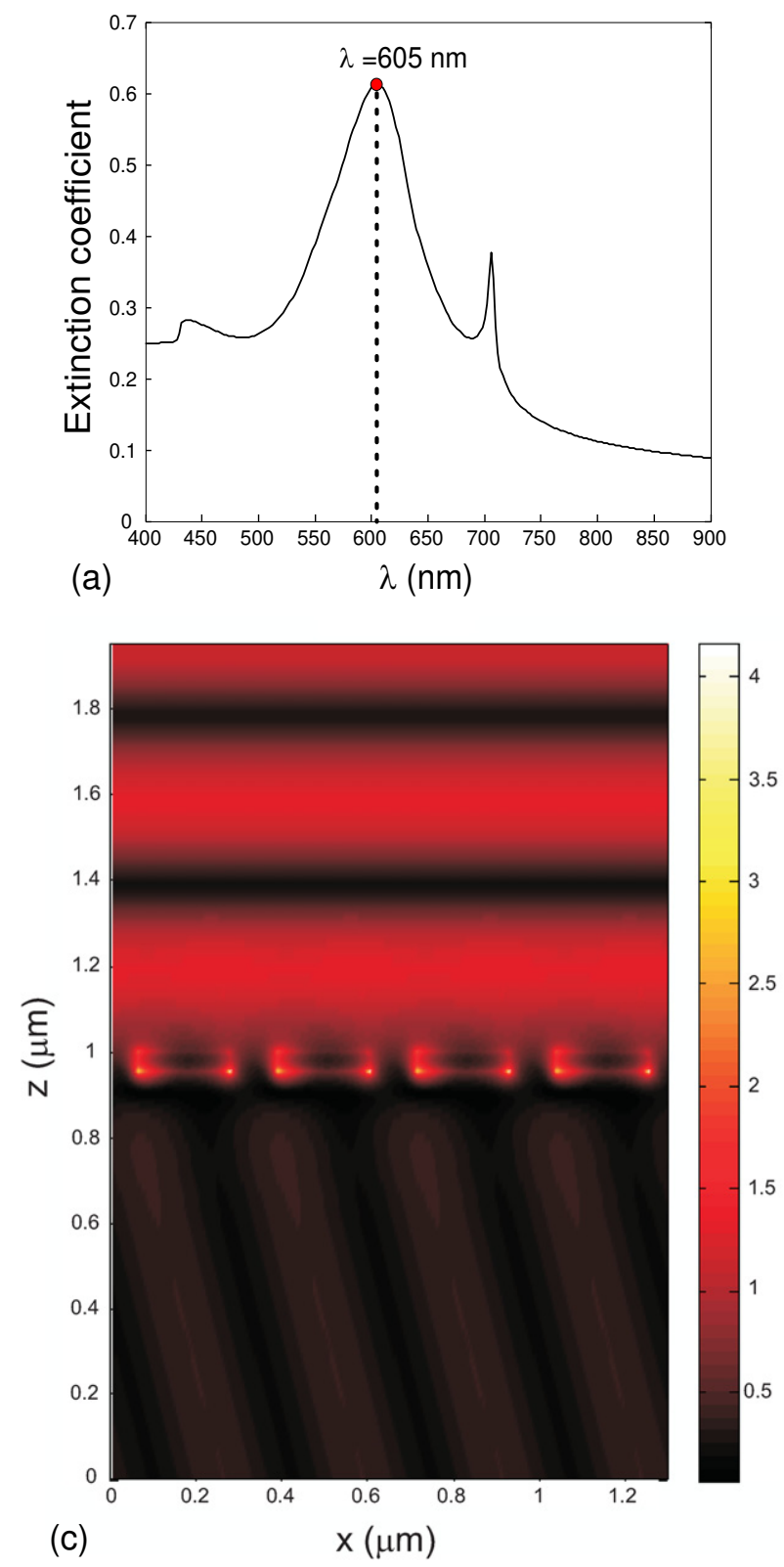
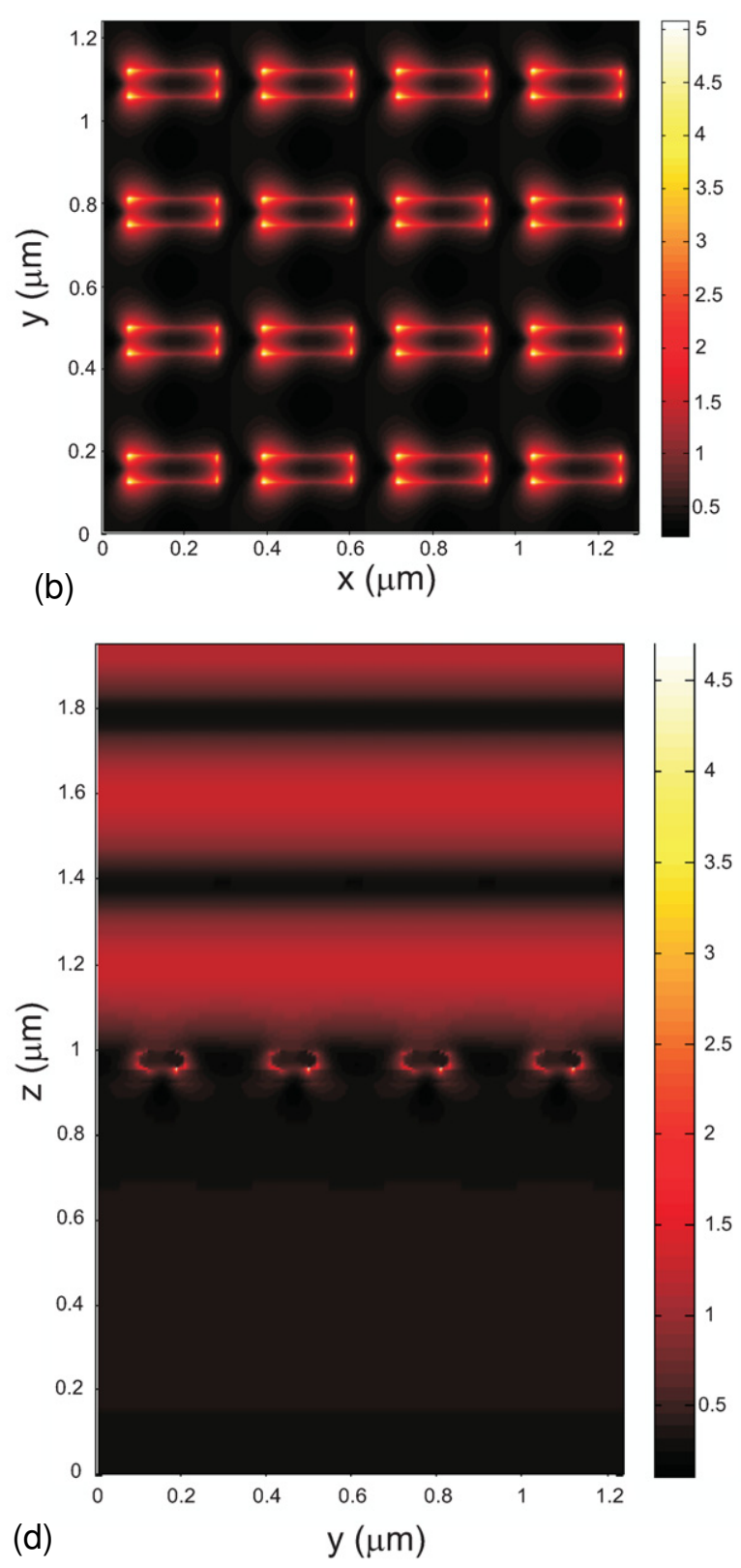

Figure 5. Electric field amplitude maps at incidence angle $\theta=40^{\circ}$ for the first peak $\lambda=605 \mathrm{~nm}(a)$ in $X Y$ plane (into ITO layer for $10 \mathrm{~nm}$ under the gold nanostructure) $(b), X Z(c)$ and $Y Z(d)$ median planes.

a modification of the electric distribution around each particle with privileged containment of light along the $Y$ polarization axis is observed.

\section{Conclusion}

To summarize, a SFM-FDTD algorithm for studying the interaction of light with an artificial periodic metallic nanostructures at oblique incidence is implemented. The present approach includes two coupled parts: the first called the SFM method which uses ingenious intermediate variables in order to solve Maxwell's equations. The second called the CP model enables calculations of the metal dispersion. We have shown that our code is able to give useful results concerning the optical response spectra and cartography field of nanostructures at oblique incidence. Finally, this original method allows performing, optimizing surface-enhanced Raman scattering for nano-optical devices as well as pointing out some recommendations for further nano-optic experiments. Let us mention that our code permits a systematic simulation study on the influence of the particles shape, the grating constant and the particles dielectric environment on the extinction spectra of metal nanoparticles arrays. Consequently, it is hoped that the present theoretical simulations will stimulate new experiments.

\section{Acknowledgments}

The authors are greatly indebted to N Félidj for his useful discussions. 

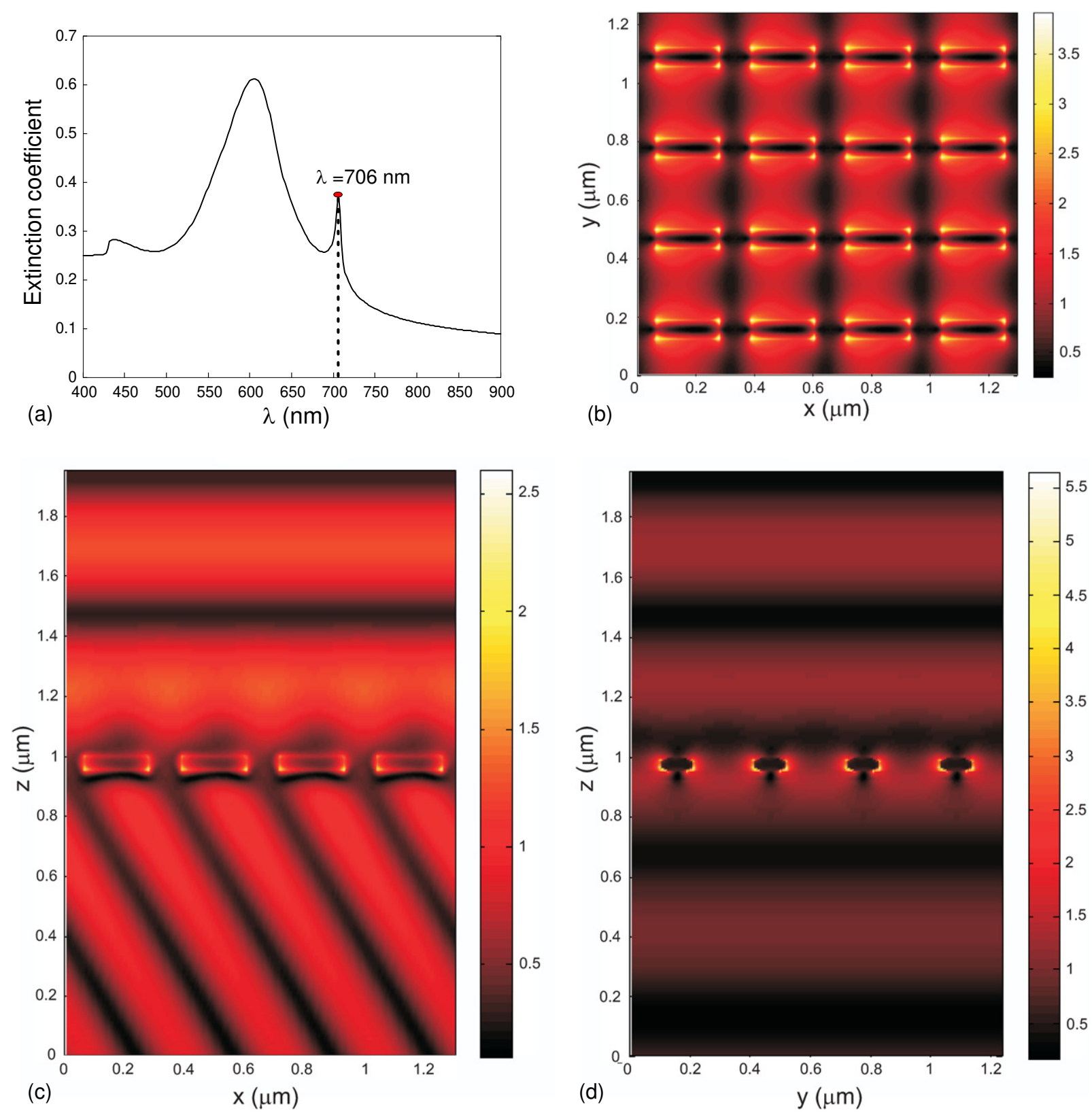

Figure 6. Electric field amplitude maps at incidence angle $\theta=40^{\circ}$ for the second peak $\lambda=706 \mathrm{~nm}(a)$ in $X Y$ plane (into ITO layer for $10 \mathrm{~nm}$ under the gold nanostructure) $(b), X Z(c)$ and $Y Z(d)$ median planes.

\section{References}

[1] Etchegoin P G, Le Ru E C and Meyer M 2006 An analytic model for the optical properties of gold J. Chem. Phys. 125164705

[2] Etchegoin P G, Le Ru E C and Meyer M 2007 An analytic model for the optical properties of gold J. Chem. Phys. 127189901 (erratum)

[3] Hao F and Nordlander P 2007 Efficient dielectric function for fdtd simulation of the optical properties of silver and gold nanoparticles Chem. Phys. Lett. 446 115-18

[4] Palik E 1985 Handbook of Optical Constants of Solids (San Diego, CA: Academic)

[5] Johnson P B and Christy R W 1972 Optical constants of the noble metals Phys. Rev. B 6 4370-79

[6] Belkhir A and Baida F I 2008 Three-dimensional finite-difference time-domain algorithm for oblique incidence with adaptation of perfectly matched layers and non-uniform meshing: application to the study of a radar dome Phys. Rev. E 77056701

[7] Baida F I and Belkhir A 2009 Split-field FDTD method for oblique incidence study of periodic dispersive metallic structures Opt. Lett. 34 2453-55

[8] Belkhir A, Arar O, Benabbes S S, Lamrous O and Baida F I 2010 Implementation of dispersion models in the split-field-finite-difference-time-domain algorithm for the study of metallic periodic structures at oblique incidence Phys. Rev. E 81046705

[9] Vial A and Laroche T 2007 Description of dispersion properties of metals by means of the critical points model and application to the study of resonant structures using the FDTD method J. Phys. D: Appl. Phys. 40 7152-58

[10] Vial A and Laroche T 2008 Comparison of gold and silver dispersion laws suitable for FDTD simulations Appl. Phys. B: Lasers Opt. 93 139-43 
[11] Vial A 2007 Implementation of the critical points model in the recursive convolution method for modelling dispersive media with the finite-difference time domain method J. Opt. A: Pure Appl. Opt. 9 745-48

[12] Veysoglu M E, Shin R T and Kong J A 1993 A finite-difference time-domain analysis of wave scattering from periodic surfaces: oblique-incidence case J. Electromagn. Waves Appl. 7 1595-607

[13] Wu B-I, Yang E, Kong J A, Oswald J A, McIntosh K A, Mahoney L and Verghese S 2000 Analysis of photonic crystal filters by the finite-difference time-domain technique Microwave Opt. Technol. Lett. 2781
[14] Félidj N, Laurent G, Aubard J, Lèvi G, Hohenau A, Krenn J R and Aussenegg F R 2005 Grating-induced plasmon mode in gold nanoparticle arrays J. Chem. Phys. 123221103

[15] Carron K T, Fluhr W, Meier M, Wokaun A and Lehmann H W 1986 Resonances of two-dimensional particle gratings in surface-enhanced raman scattering J. Opt. Soc. Am. B 3430

[16] Meier M, Wokaun A and Liao P F 1985 Enhanced fields on rough surfaces: dipolar interactions among particles of sizes exceeding the rayleigh limit J. Opt. Soc. Am. B 2932

[17] Lamprecht B, Schider G, Lechner R T, Ditlbacher H, Krenn J R, Leitner A and Aussenegg F R 2000 Metal nanoparticle gratings: Influence of dipolar particle interaction on the plasmon resonance Phys. Rev. Lett. 844721 